\title{
ACTITUDES DEL PROFESORADO DE CONSERVATORIO SOBRE LA INTEGRACIÓN EDUCATIVA: UN ANÁLISIS EXPLORATORIO.
}

\author{
CONSERVATORY TEACHING STAFF'S ATTITUDES ON THE EDUCATIVE \\ INTEGRATION: AN EXPLORATORY ANALYSIS
}

\author{
Leandro Navas Martínez* y Ana Isabel Castillo Martínez** \\ Universidad de Alicante
}

\section{RESUMEN}

Los objetivos de este estudio son conocer las actitudes del profesorado de Conservatorio ante los Alumnos con Necesidades Educativas Especiales (a.c.n.e.e.) y ante la integración, así como establecer posibles parámetros que fundamenten tales actitudes. Docentes de Conservatorio $(n=42)$ con una edad media de 34,7 años $(\sigma=7,24)$, responden a un cuestionario de actitudes (Mula et al., 2002) abreviado y reformulado según los fines perseguidos. Se realizan análisis factoriales cuyos resultados muestran que hay 3 factores de segundo orden subyacentes a las actitudes de los profesores y que suponen una polarización de las actitudes ante la integración (actitud negativa y posición positiva).

Palabras clave: actitudes docentes, programa de integración, enseñanza musical, necesidades educativas especiales.

\section{ABSTRACT}

We think it is essential to bf the attitudes of teaching staff towards students with special educational needs and their integration in musical education, and how to establish parameters in which we can base those attitudes. 42 teaching staff of the Conservatory $(n=42)$ with an average age of 34,7 years old $(\sigma=7,24)$, answer an attitudes questionnaire (Mula et al., 2002) abbreviated and reformulated ac-

*. Leandro Navas Martínez. Catedrático de E.U. de Psicología Evolutiva y de la Educación. Ha desempeñado los cargos de Vicedecano de Prácticas y de Secretario de la Facultad de Educación de la Universidad de Alicante. Sus líneas de investigación giran en torno a los determinantes del rendimiento académico, la motivación, la evaluación educativa y la evaluación de programas educativos. Ha dirigido y participado en varios proyectos subvencionados por el CIDE, en proyectos I+D y en investigaciones subvencionadas por el Ayuntamiento de Alicante. E-mail: Leandro.Navas@ua.es

**. Ana Isabel Castillo Martínez. Profesora Superior de Piano por el Conservatorio de Alicante. Realiza estudios de tercer ciclo en el Departamento de Didáctica General y Didácticas Específicas de la Facultad de Educación de la Universidad de Alicante. 
cording to the goals pursved. Factorial analyses are carried out with results that show 3 factors of second order related to the attitudes of the teachers which imply a positive position about the integration, and a negative position towards it.

Key words: educational attitudes, integration program, musical education, special educational needs.

\section{Introducción}

El Real Decreto 334/1985 de la Educación Especial inicia la integración escolar en España en Infantil (a la sazón Preescolar) y Primaria (entonces E.G.B.), pero este programa no afectó a las enseñanzas musicales. Por otra parte, la enseñanza en los Conservatorios ha cambiado en los últimos años. El Libro Blanco (1989) para la Reforma del Sistema Educativo propone la regulación de las enseñanzas artísticas dentro de una reforma global del Sistema Educativo y en la Ley Orgánica 1/1990 de Ordenación General del Sistema Educativo (L.O.G.S.E.), artículos 42.3 y 39.5 , se da forma jurídica a lo propuesto. Esta ley introduce, también, los conceptos de necesidades educativas especiales y de normalización y extiende la integración a todo el sistema educativo. En consecuencia, esta nueva concepción comprende a todas las enseñanzas y a todos los centros educativos (incluidos los Conservatorios de Música).

La atención a la diversidad asume que hay alumnos (a.c.n.e.e.) que presentan dificultades mayores que el resto de sus iguales para acceder a los contenidos establecidos en el currículo y que precisan ayudas psicoeducativas diferenciadas y específicas (Blanco, 1992; Warnock, 1978). La normalización (Nirje, 1970) implica ofrecer a las personas con discapacidad unas condiciones de vida lo más parecidas a las del resto de ciudadanos. Y el desarrollo de la inclusión conlleva cambios en el sistema, en las políticas educativas, en el funcionamiento de las escuelas y en las prácticas docentes. La inclusión trata de educar con éxito a la diversidad del alumnado para anular la desigualdad, ya que esta convivencia y el aprendizaje en grupo benefician a todos los alumnos (Arnaiz, 2005). Para una enseñanza inclusiva es necesario que el profesorado tenga una formación continua para conocer mejor la diversidad del alumnado, tener una percepción positiva de los a.c.n.e.e., y para poder desarrollar habilidades docentes que ayuden a esos alumnos a aprender al lado de sus iguales (Marchesi, 1999a). La formación permanente del docente y el apoyo de los profesores especializados contribuye al avance de la integración educativa.

Así, si la enseñanza, en general, asume las nociones de atención a la diversidad, de integración, de enseñanza adaptativa (Wang, 1980, 1995) y de inclusión (Ainscow, 1995), la enseñanza de la música no debe quedar al margen. En ese sentido, lo que puede plantearse es cuáles son las actitudes de los docentes de los Conservatorios ante la integración y ante los a.c.n.e.e. Ante el programa de integración, probablemente la actitud sea positiva o de indiferencia, dado el modo como se ha integrado este tipo de alumnado en los Conservatorios, que la puesta en marcha del programa se ha realizado sin orientaciones claras, que, a diferencia de otros centros, los Conservatorios no cuentan con equipos especializados (psicopedagogos y especialistas de apoyo) y que, al impartir enseñanzas no obligatorias, el equipo de orientación escolar del sector da prioridad a la asistencia al alumnado que cursa enseñanzas obligatorias. Tampoco se llevan a cabo cursos de formación para el profesorado de música en atención a la diversidad, en atención a los a.c.n.e.e. y en adaptaciones curriculares en Conservatorios. 
Leandro Navas Martínez et al. Actitudes del profesorado de conservatorio sobre la integración...

En cuanto a las actitudes del profesorado hacia los a.c.n.e.e., puede ocurrir que un elevado número de docentes del Conservatorio considere que, en estos centros, sólo deben estudiar alumnos con unas capacidades musicales especiales (así se enuncia en los preliminares del currículo de grado elemental y medio: estas enseñanzas dotarán a los alumnos de suficientes capacidades como para acceder a la especialización musical en el grado superior). Sin embargo, el alumno que comienza estudios de música no está obligado a hacer carrera superior en ella, todos los alumnos tienen derecho a una educación en cualquier centro educativo sostenido con fondos públicos y pueden decidir ingresar en un Conservatorio o en una Escuela de música, sin tener que ser discriminados por no tener una idea clara de lo que estudiarán en el futuro o por precisar una respuesta educativa especial.

En suma, como se han realizado pocas investigaciones acerca de las actitudes del profesorado de los Conservatorios y la enseñanza de la música no se ha visto favorecida por estudios teóricos profundos (Fuentes y Cervera, 1989), cobra sentido esta investigación cuyos objetivos son: Conocer las actitudes del profesorado de los Conservatorios ante los a.c.n.e.e. y ante la integración, y establecer algunos posibles parámetros en los que fundamentar tales actitudes.

\section{Método}

\section{Participantes}

Participan 42 docentes de grado elemental, medio y superior de los Conservatorios de la provincia de Alicante (Torrevieja, Alicante y Pilar de la Horadada) y del Conservatorio de Almería, con edades comprendidas entre los 25 y los 51 años y una edad media de 34,71 $(\sigma=7,24)$. El muestreo es incidental. El $69 \%$ son hombres y el resto mujeres. En cuanto al nivel docente, el 16,7\% ejerce en grado elemental, el $69 \%$ en grado medio y el $14,3 \%$ restante en grado superior.

\section{Instrumento}

Se emplean las preguntas escalares de un cuestionario más amplio elaborado en otra investigación sobre un tema similar (Mula et al., 2002) que van destinadas a valorar las actitudes de los docentes ante la integración y ante los a.c.n.e.e. (ver tabla 1). Las escalas son de cinco puntos y varían desde "Muy en Desacuerdo" ("MD") hasta "Totalmente de Acuerdo" ("TA").

\section{Variables}

Se consideran como variables directamente observadas las respuestas a las preguntas seleccionadas del Cuestionario de Mula et al. (2002) que se muestran en la tabla 1. Las variables latentes son las que resultan de los análisis exploratorios (ver sección de resultados). 
1: Texto de las preguntas seleccionadas del Cuestionario de Actitudes (Mula et al., 2002).

\section{BLOQUE I}

1. El reto que representa estar en una clase ordinaria del conservatorio estimula el desarrollo académico del a.c.n.e.e.

2. La formación sobre la temática de los a.c.n.e.e. atañe a todos los profesores del conservatorio.

3. Es difícil mantener el orden y la disciplina en un clase ordinaria en la que haya a.c.n.e.e.

4. La integración supone una interacción en grupo que favorece la comprensión y aceptación de la diferencia.

5. En el fondo la integración tiene un carácter más político o legal que educativo.

6. El a.c.n.e.e. no puede enfrentarse a los desafíos que plantean las clases del conservatorio en igualdad de condiciones.

7. El proceso de integración está siendo más impuesto que consultado.

8. La integración favorece la preparación de los alumnos para vivir en una sociedad integrada.

\section{BLOQUE II}

1. La atención extra que requieren los a.c.n.e.e. irá en detrimento de los otros alumnos.

2. El a.c.n.e.e. tendrá un mejor desarrollo de sus capacidades musicales en una clase ordinaria.

3. La integración del a.c.n.e.e. promoverá su independencia social.

4. La integración tiene un efecto negativo sobre el desarrollo emocional del a.c.n.e.e.

5. Los a.c.n.e.e. se comportarán de forma apropiada en clases ordinarias.

6. La valoración social de los a.c.n.e.e., tal como la percibe el resto de compañeros, mejora a través de la interacción con el grupo.

7. En general, la integración es una práctica educativa deseable.

8. Es difícil mantener el orden y la disciplina en una clase de música a la que asisten a.c.n.e.e.

9. A los a.c.n.e.e. no les preocupa demasiado ser diferentes, a no ser que les haga competir con los demás alumnos.

\section{Procedimiento}

Tras solicitar las oportunas autorizaciones a los directores de los Conservatorios, se distribuyó el cuestionario entre el profesorado que lo respondió voluntariamente. Se garantizó la confidencialidad de las respuestas.

\section{Diseño}

Dado que los sujetos no son escogidos al azar y que no se manipulan las variables, se trata de un diseño correlacional. Los datos se tratan garantizando el anonimato y la intimidad de los sujetos. 
Leandro Navas Martínez et al. Actitudes del profesorado de conservatorio sobre la integración...

\section{Análisis de datos}

Con las respuestas al cuestionario se realizan análisis factoriales exploratorios con el método de extracción de componentes principales y, al buscar la mayor independencia posible entre los factores, el método de rotación Varimax. Se usa el programa SPSS (versión 11.5 para Windons). El análisis factorial requiere que las variables tengan una distribución normal en la población. No se realizan pruebas (Chi-cuadrado o Kolgomorov-Smirnov) para comprobar este supuesto porque, teniendo en cuenta el tamaño de la muestra $(n>30)$, asumimos la normalidad de la distribución ya que: "Una gran mayoría de las características psicológicas tienen una distribución normal en la población" (Seoane, Rechea y Diges, 1982, p. 430). Las actitudes, obviamente, son variables de tipo psicológico.

\section{Resultados}

En primer lugar, se someten a análisis factorial las respuestas a las ocho cuestiones del primer bloque. La pertinencia de este análisis se confirma con la prueba de esfericidad de Barlett que resulta estadísticamente significativa $\left(\chi_{(28)}^{2}=68,180 ; p<0,001\right)$, lo que permite rechazar la hipótesis de independencia entre las variables. La rotación converge en 4 iteraciones y surgen 3 factores que explican el 65,69\% de la varianza. En la tabla 2 se resume la matriz de componentes rotados o solución factorial rotada.

TABLA 2: Matriz factorial rotada que surge tras realizar el análisis factorial (extracción por componentes principales y rotación Varimax) con las respuestas a las preguntas del Bloque I.

\begin{tabular}{|l|c|c|c|}
\hline \multicolumn{1}{|c|}{ Ítems } & Factor I & Factor II & Factor III \\
\hline $\begin{array}{l}\text { 1. ... estar en una clase ordinaria ... estimula el desarrollo } \\
\text { académico del a.c.n.e.e. }\end{array}$ & 0,707 & & \\
$\begin{array}{l}\text { 2. La formación sobre ... a.c.n.e.e. atañe a todos los profeso- } \\
\text { res del Conservatorio }\end{array}$ & 0,625 & \\
$\begin{array}{l}\text { 4. .. supone una interacción ... la comprensión y aceptación } \\
\text { de diferencia }\end{array}$ & 0,805 & \\
$\begin{array}{l}\text { 8. .. favorece la preparación de los alumnos para vivir en una } \\
\text { sociedad ... }\end{array}$ & 0,717 & 0,814 & \\
\hline $\begin{array}{l}\text { 3. Es difícil mantener el orden y la disciplina ... que haya } \\
\text { a.c.n.e.e. }\end{array}$ & & 0,711 & \\
$\begin{array}{l}\text { 5. ... tiene un carácter más político legal que educativo } \\
\text { 6. El a.c.n.e.e. no puede enfrentarse a los desafíos ... en } \\
\text { igualdad de condiciones }\end{array}$ & & 0,660 & \\
\hline 7. ... más impuesto que consultado & & & 0,842 \\
\hline
\end{tabular}

Factor I: Actitud positiva; Factor II: Actitud reactiva, y Factor III: Actitud frente a la imposición.

En el primer factor presentan la mayor carga factorial las preguntas 1, 2, 4 y 8 que hacen referencia a actitudes positivas hacia la integración, con enunciados como "El reto que representa estar en una clase ordinaria estimula el desarrollo académico del a.c.n.e.e." y "La 
integración favorece la preparación de los alumnos para vivir en una sociedad integrada". Denominamos a este primer factor "Actitud positiva". Da cuenta del 30,68\% de la varianza $\mathrm{y}$ tiene una fiabilidad (consistencia interna alfa de Cronbach) $\alpha=0,69$.

El segundo factor explica el $21,53 \%$ de la varianza y la mayor carga factorial la muestran las preguntas 3, 5 y 6 con expresiones como "Es difícil mantener el orden y la disciplina en una clase ordinaria en la que haya a.c.n.e.e" y "El a.c.n.e.e. no puede enfrentarse a los desafíos que plantean las clases del Conservatorio en igualdad de condiciones". Este factor manifiesta actitudes de rechazo ante la integración y lo hemos nombrado "Actitudes reactivas". La fiabilidad (consistencia interna) es $\alpha=0,66$.

El tercer factor justifica el 13,38\% de la varianza. La mayor carga factorial es la de la pregunta 7 que expresa la idea "El proceso de integración está siendo más impuesto que consultado". Se designa "Actitud frente a la imposición". La consistencia interna no es posible calcularla al estar formado el factor por un ítem.

En segundo lugar se realiza un análisis factorial con las respuestas a las 9 preguntas del Bloque II. La prueba de esfericidad de Barlett es estadísticamente significativa $\left(\chi_{(36)}^{2}=100,952 ; p<0,001\right)$. La rotación converge en 6 iteraciones dando lugar a 4 factores que explican el $75,30 \%$ de la varianza. En la tabla 3 se resume la matriz factorial rotada.

TABLA 3: atriz factorial rotada que surge tras realizar el análisis factorial (extracción por componentes principales y rotación Varimax) con las respuestas a las preguntas del Bloque II.

\begin{tabular}{|c|c|c|c|c|}
\hline Ítems & Factor I & Factor II & Factor III & Factor IV \\
\hline $\begin{array}{l}\text { 4. ... tiene un efecto negativo sobre el desarrollo } \\
\text { emocional... } \\
\text { 6. La valoración social ... mejora a través de la } \\
\text { interacción en grupo } \\
\text { 9. A los a.c.n.e.e. no les preocupa demasiado } \\
\text { ser diferentes ... }\end{array}$ & $\begin{array}{r}0,778 \\
-0,792 \\
0,668\end{array}$ & & & \\
\hline $\begin{array}{l}\text { 2. El a.c.n.e.e. tendrá un mejor desarrollo de } \\
\text { sus capacidades ... } \\
\text { 5. Los a.c.n.e.e. se comportarán de forma apro- } \\
\text { piada en clases ordinarias }\end{array}$ & & $\begin{array}{l}0,882 \\
0,836\end{array}$ & & \\
\hline $\begin{array}{l}\text { 3. La integración del a.c.n.e.e. promoverá su in- } \\
\text { dependencia social } \\
\text { 7. En general, la integración es una práctica } \\
\text { educativa deseable }\end{array}$ & & & $\begin{array}{l}0,926 \\
0,692\end{array}$ & \\
\hline $\begin{array}{l}\text { 1. La atención extra que requieren ... irá en de- } \\
\text { trimento de los otros ... } \\
\text { 8. Es difícil mantener el orden y la disciplina ... } \\
\text { asisten a.c.n.e.e. }\end{array}$ & & & & $\begin{array}{l}0,918 \\
0,705\end{array}$ \\
\hline
\end{tabular}

Factor I: Orientación negativa; Factor II: Orientación favorable; Factor III: Integración positiva, y Factor IV: Distorsión.

El primer factor explica el $31,58 \%$ de la varianza y presentan la mayor carga factorial las cuestiones 4, 6 y 9, con enunciados del tipo "La integración tiene un efecto negativo 
sobre el desarrollo emocional del a.c.n.e.e.” y "A los a.c.n.e.e. no les preocupa ser diferentes, a no ser que se les haga competir con los demás alumnos". Se da a este factor el nombre de "Orientación negativa" porque estas expresiones implican que la integración tiene consecuencias negativas para los a.c.n.e.e. Tiene una fiabilidad (consistencia interna) $\alpha=0,70$.

El segundo factor da cuenta del 19,49\% de la varianza. En él saturan los elementos 2 y 5 con expresiones como "El a.c.n.e.e. tendrá un mejor desarrollo de sus capacidades musicales en una clase ordinaria" y "Los a.c.n.e.e. se comportarán de forma apropiada en clases ordinarias". Lo denominamos "Orientación favorable" dado que indica ventajas de la integración para los a.c.n.e.e. La fiabilidad es $\alpha=0,69$.

En el tercer factor, que explica el $12,52 \%$ de la varianza, presentan la mayor carga factorial las cuestiones 3 y 7 que expresan ideas como "La integración del a.c.n.e.e. promoverá su independencia social" y "En general, la integración es una práctica educativa deseable". Como indica que la integración es positiva para los a.c.n.e.e. lo hemos designado "Integración positiva" y presenta un $\alpha=0,69$.

El cuarto factor justifica el $11,71 \%$ de la varianza y viene a significar que el programa de integración perjudica a los alumnos sin necesidades educativas especiales, por lo que se le da el nombre de "Distorsión". La mayor saturación la presentan los elementos 1 y 8 con expresiones del tipo "La atención extra que requieren los a.c.n.e.e. irá en detrimento de los otros alumnos" y "Es difícil mantener el orden y la disciplina en una clase de música a la que asisten a.c.n.e.e.". Tiene un $\alpha=0,67$.

Por último, se realiza un análisis factorial de segundo orden sobre los factores obtenidos en los análisis anteriores. La matriz factorial rotada (ver la tabla 4) converge en 4 iteraciones dando lugar a tres factores que explican el $65,27 \%$ de la varianza con una fiabilidad $\alpha=0,66$.

TABLA 4: Matriz factorial rotada que surge tras realizar el análisis factorial de segundo orden (extracción por componentes principales y rotación Varimax) con los factores de primer orden obtenidos.

\begin{tabular}{|l|c|c|c|}
\hline \multicolumn{1}{|c|}{ Factores de Primer orden } & Factor I & Factor II & Factor III \\
\hline Actitud positiva & 0,821 & & \\
Integración positiva & 0,860 & & \\
\hline Actitud frente a la imposición & & 0,770 & \\
Orientación favorable & & $-0,377$ & \\
Distorsión & & 0,762 & \\
\hline Actitud reactiva & & & 0,822 \\
Orientación negativa & & & 0,817 \\
\hline
\end{tabular}

Factor I: "Postura positiva ante la integración del a.c.n.e.e.". Factor II: "Actitud negativa ante el programa de integración". Factor III: "Postura negativa ante la integración del a.c.n.e.e.".

El primer factor de segundo orden da cuenta del 25,22\% de la varianza. En él presentan las saturaciones más elevadas los factores de primer orden denominados "Actitud positiva" e "Integración positiva". Hemos llamado a este factor de segundo orden "Postura positiva 
ante la integración del a.c.n.e.e." ya que engloba una actitud general positiva hacia el programa de integración y hacia los a.c.n.e.e. La consistencia interna es $\alpha=0,68$.

En el segundo factor de segundo orden saturan los factores de primer orden "Distorsión", "Actitud frente a la imposición" y, con carga negativa, "Orientación favorable". Este factor justifica el $22,098 \%$ de la varianza y representa una postura negativa del docente ante el a.c.n.e.e. y la creencia de que el programa de integración puede afectar negativamente a los alumnos. Lo hemos denominado "Actitud negativa ante el programa de integración". $\mathrm{Su}$ consistencia interna es $\alpha=0,15$, que asciende a 0,49 si se suprime el factor de primer orden "Orientación favorable". Considerando que la carga de este factor es negativa y no muy elevada $(-0,377)$ y que la fiabilidad aumenta al ser suprimido, no se tiene en cuenta (ver figura 1).

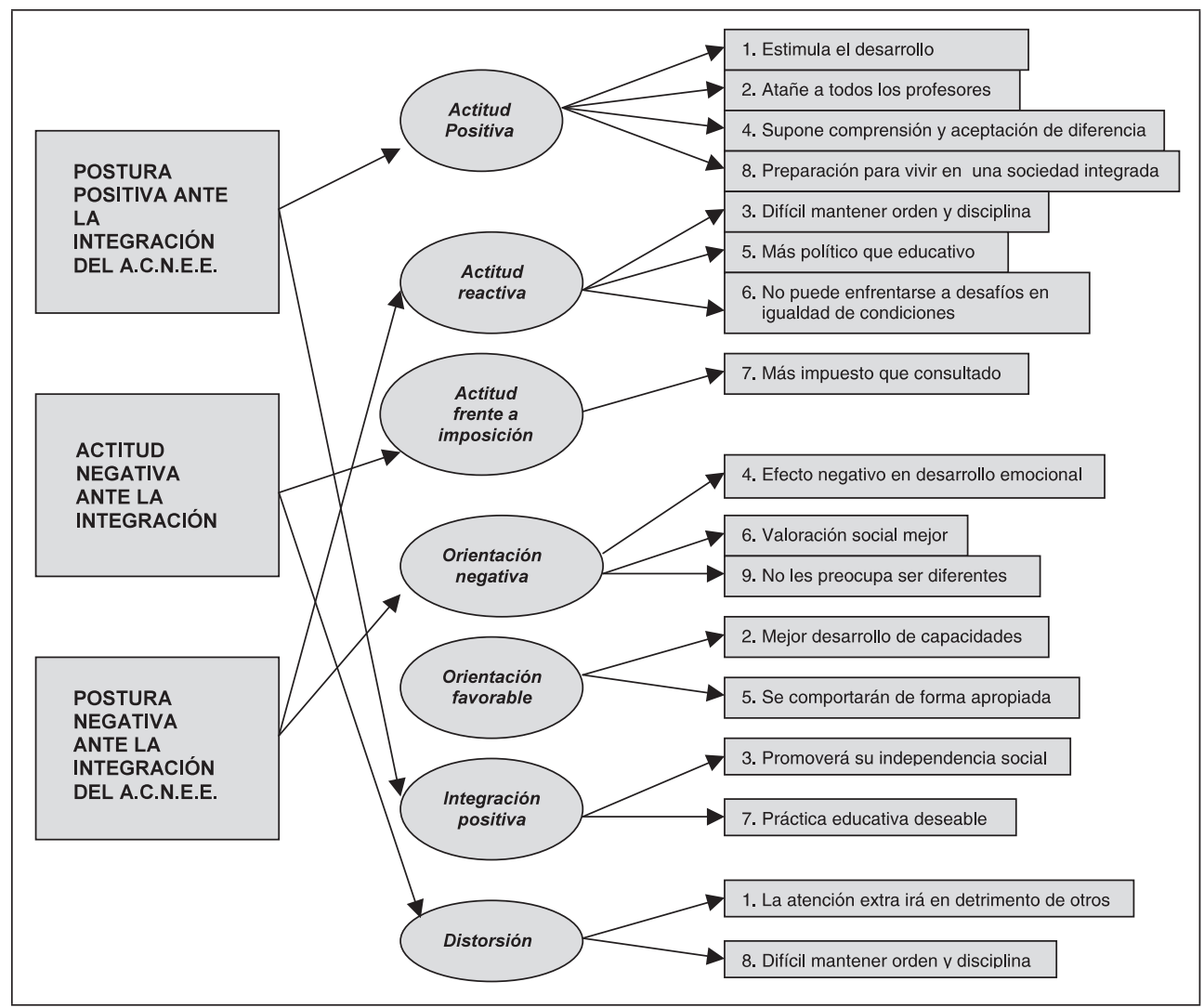

FIGURA 1.

Variables observadas y variables latentes de primer y segundo orden.

El tercer factor de segundo orden explica el 17,96\% de la varianza. Muestran las saturaciones factoriales más elevadas los factores de primer orden "Actitud reactiva" y "Orienta- 
ción negativa". Hemos designado a este factor "Postura negativa ante la integración del a. c.n.e.e." porque representa la percepción del docente ante la capacidad de aprendizaje del a.c.n.e.e. Su consistencia interna es $\alpha=0,58$.

Las estructuras o dimensiones latentes en las que se basan las actitudes del profesorado de los Conservatorios, a partir de las variables directamente observadas, quedan resumidas en la figura 1.

\section{Conclusiones}

Los objetivos de este trabajo eran conocer las actitudes del profesorado de los Conservatorios de música ante la integración y ante los a.c.n.e.e. y establecer los parámetros en los que se asientan esas actitudes.

En primer lugar, en lo que concierne a las actitudes ante la integración, surgen tres variables latentes. Una actitud favorable y comprensiva, "Actitud positiva", una "Actitud reactiva" a la que subyacen opiniones de rechazo al programa de integración, y "Actitud frente a la imposición" que refleja la percepción del profesorado de que la integración ha sido impuesta (a pesar de los Cuadernos para el debate publicados por la Administración Educativa). Estos resultados reafirman los de Mula et al. (2002) en cuanto a la existencia de una actitud positiva, de una actitud reactiva y de una polarización en esas actitudes docentes sobre la integración. Como señala Marchesi (1999a), la integración "no significa simplemente escolarizar a los alumnos en los centros ordinarios, sino que exige un cambio en las escuelas" (p. 34). Entendemos que el cambio que el Conservatorio precisa afecta al nivel de preparación de sus docentes y a los recursos y ayudas que puedan recibir de otros profesionales. Para los docentes del Conservatorio la integración es un programa novedoso y no es de extrañar que, como establecen otros estudios (Bowman, 1986; Marchesi, 1999b; Navas, L., Torregrosa, G., Alaminos, A. y Mula, A., 2001; Vlachou y Barton, 1994; Yola y Ward, 1987) ajenos a la educación musical, el profesorado muestre cierta resistencia a incorporarse al mismo, quizás porque no se siente preparado y porque percibe falta de apoyos $\mathrm{y}$ de recursos.

En relación con las actitudes de los profesores ante los a.c.n.e.e., se aprecia la existencia de cuatro variables no directamente observadas. Una "Orientación negativa" que expresa las consecuencias negativas de integrar a los a.c.n.e.e. en aulas ordinarias. Una "Orientación favorable" relacionada con ventajas que los profesores ven en la integración de los a. c.n.e.e. (desarrollar mejor sus capacidades musicales o portarse bien). La "Integración positiva" que refleja las actitudes favorables frente a la integración de los a.c.n.e.e. Y, finalmente, "Distorsión" que representa los perjuicios que supuestamente tiene la integración para alumnos sin necesidades educativas especiales. Vuelve a apreciarse, así, una polarización en las actitudes del profesorado.

En un mayor nivel de generalidad aparecen, según el análisis factorial de segundo orden, tres variables latentes. La "Postura positiva ante la integración del a.c.n.e.e." viene de resumir la variabilidad común entre "Actitud positiva" e "Integración positiva". La "Actitud negativa ante el programa de integración" asume la comunalidad presente en "Actitud frente a la imposición" y "Distorsión". Y finalmente, la "Postura negativa ante la integración del a.c.n.e.e." subsume a la "Actitud reactiva" y a la "Orientación negativa". 
En todo caso, ejerciendo la autocrítica, debemos considerar las limitaciones de la técnica de recogida de datos empleada (los participantes pueden introducir sesgos en función de lo socialmente deseable o de lo que se desea decir sobre los propios pensamientos).

En resumen, a tenor de los resultados obtenidos, consideramos que hay que intentar cambiar esa polarización que hemos detectado en las actitudes de los profesores del Conservatorio sobre la integración y los a.c.n.e.e. Urge variar las actitudes poco favorables e incrementar las actitudes positivas y, de cara a investigaciones futuras, procede ampliar la muestra y realizar análisis confirmatorios. Cambiar las actitudes de los docentes es un reto y, a la vez, una vía de trabajo que queda abierta.

\section{Referencias bibliográficas}

Ainscow, M. (1995). Necesidades especiales en el aula. Guía para la formación del profesorado. Madrid: Narcea/UNESCO.

Arnaiz, P. (2005). "La programación de aula". En P. Arnaiz (Ed.), Atención a la diversidad. Programación curricular (pp. 117-144). San José de Costa Rica: Universidad Estatal a Distancia.

Blanco, R. (Dir.) (1992). Alumnos con necesidades educativas especiales y adaptaciones curriculares. Madrid: Dirección General de Renovación Pedagógica.

Bowman, I. (1986). "Teacher training and the integration of handicapped pupils: Some findings from a fourteen nation Unesco study". European Journal of Special Needs Education, 1 (1), 29-38.

Fuentes, P. y Cervera, J. (1989). Pedagogía y didáctica para músicos. Valencia: Piles.

Marchesi, A. (1999a). "La práctica de las escuelas inclusivas". En A. Marchesi, C. Coll y J. Palacios (Comps.), Desarrollo psicológico y educación 3. Trastornos del desarrollo y necesidades educativas especiales (pp. 45-78). Madrid: Alianza.

Marchesi, A. (1999b). "Del lenguaje de la deficiencia a las escuelas inclusivas". En A. Marchesi, C. Coll y J. Palacios (Comps.), Desarrollo psicológico y educación 3. Trastornos del desarrollo y necesidades educativas especiales (pp. 21-43). Madrid: Alianza.

MEC (1989). Libro blanco para la reforma del sistema educativo. Madrid: Secretaría General de Educación.

MEC (1985). Real Decreto, 334/1985, de 6 de Marzo, de Ordenación de la Educación Especial. (B. O.E., 16-3-1985).

MEC (1990). Ley Orgánica 1/1990, de 3 de octubre, de Ordenación General del Sistema Educativo. (B.O.E., 4-10-1990).

Mula, A., Alaminos, A., Navas, L., Torregrosa, G., Sabroso, A., López-Cano, D., Mora, M. J., Sánchez, J. L., Panera, I. y Santacreu, O. (2002). Incidencia de las actitudes y expectativas de alumnos y profesores sobre el desarrollo del programa de integración. Alicante: Universidad de Alicante.

Navas, L., Torregrosa, G., Alaminos, A. y Mula, A. (2001). "Un análisis exploratorio de las actitudes del profesorado ante la integración". En el I Congreso Nacional de Atención a la Diversidad. Elche.

Nirje, B. (1970). "The normalization principle, implications and comments". Symposium sobre normalización. Madrid.

Seoane, J., Rechea, C., y Diges, M. (1982). “Conceptos básicos de inferencia estadística”. En J. Seoane (Dir.), Psicología matemática I (pp. 419-430). Madrid: U.N.E.D. 
Vlachou, A. y Barton, L. (1994). "Inclusive education: teachers and the changing cultura of schooling”. British Journal of Special Education, 21 (3), 105-107.

Wang, M. C. (1995). Atención a la diversidad del alumnado. Madrid: Narcea.

Wang, M. C. (1980). "Adaptive instruction: Building on diversity”. Theory Into Practice, 19, 122-128.

Warnock, H. M. (1978). Special education needs. Report of the commitee of Enquiry into the informe of Handicapped children and young people. Londres: Her Majesty's Office. [Trad. Revista de Educación. Número extraordinario, 1987 (pp. 44-73). Madrid: Ministerio de Educación y Ciencia].

Yola, C. y Ward, J. (1987). "Teacher's attitudes towards the integration of disabled children in regular schools". The Exceptional Child, 34 (1), 41-55.

Fecha de recepción:10-11-05

Fecha de revisión: 05-03-07

Fecha de aceptación: 08-05-07 
